\title{
Porogen Effect of Bioactive Glass on Poly(L-lactide) Scaffolds: Evidences by Electron Microscopy
}

\author{
N. B. Barroca, ${ }^{*}$ A. L. Daniel-da-Silva, ${ }^{*}$ M. H. V. Fernandes ${ }^{*}$ and P. M. Vilarinho ${ }^{*}$ \\ *Department of Ceramics and Glass Engineering, CICECO, University of Aveiro, Campus \\ Universitário de Santiago, Aveiro, 3810-193, Portugal
}

Recently, porous polymer-ceramic composites have been developed and represent promising scaffolds to be used as synthetic extracellular matrix in bone tissue engineering since they combine the advantages of these two types of materials. On the other hand bioactive glasses (BG) have been used as ceramic fillers to promote bioactivity and to enhance mechanical properties [1] and osteoblast functions [2]. Among all the requirements, these $3 \mathrm{D}$ porous structures should have a controllable average pore size larger than $100 \mu \mathrm{m}$ as well as good pore interconnectivity to allow vascularization and tissue ingrowth. The goal of this study is to investigate the effect of the addition of a bioactive glass on the porous structure development of the scaffolds prepared by thermally induced phase-separation and also to test the bioactivity of these composite scaffolds. Poly (Llactic) acid (PLLA) was chosen as the polymer matrix because of its well-known biocompatibility and adjustable physical and mechanical properties. Micron-sized $(<10 \mu \mathrm{m})$ glass from the $3 \mathrm{CaO} . \mathrm{P}_{2} \mathrm{O}_{5}-\mathrm{MgO}-\mathrm{SiO}_{2}$ system was produced in our laboratory and used as the bioactive ceramic filler.

PLLA/BG scaffolds were prepared by phase separation of 5,5\%(w/v)PLLA / 1,4dioxane / water solutions with different BG content $(0-50 \mathrm{wt} \%)$. Bioactivity test was conducted by immersion of the composites scaffolds in simulated body fluid for different periods of time $(1,4,7,14$ and 21days).

Scanning electron microscopy (SEM) was used to follow the porous structure development of the scaffolds. A clear increase of the pore size from 30 to $150 \mu \mathrm{m}$ with increasing BG content was observed (Fig. 1). This surprising effect is attributed to the large polymer exclusion during phase separation due to the possible self hydration of cations released from the glass. SEM also allows proving the formation of an apatite layer in composite scaffolds. Rod-shaped crystals organized in typical "cauliflower" morphologies can be observed on PLLA/50\%BG composites after 4 days of immersion in SBF (Fig. 2.). Fourier transform infrared spectroscopy (FTIR) spectra show a divided peak between 600 and $500 \mathrm{~cm}^{-1}$ related with the $\mathrm{P}-\mathrm{O}$ bending vibration confirming the presence of a crystalline phosphate calcium layer (Fig. 3.).

Scanning electron microscopy showed to be a very convenient technique to follow the development of porous scaffolds for biomedical applications. SEM was used here to evidence the porogen effect of bioactive glass in PLLA/BG composite scaffolds and to show the ability of these composite scaffolds to induce the "in vitro" precipitation of apatite in synthetic plasma, so far believed as the key requirement for their "in vivo" bone bonding.

References:

[1] K. Zhang, Y. Wang, M. A. Hillmyer, L. F. Francis, Biomaterials 25 (2004) 2489.

[2] H. H. Lu, A. Tang, S. C. Oh, Jeffrey P. Spalazzi, Kathie Dionisio, Biomaterials 26 (2005) 6323. 

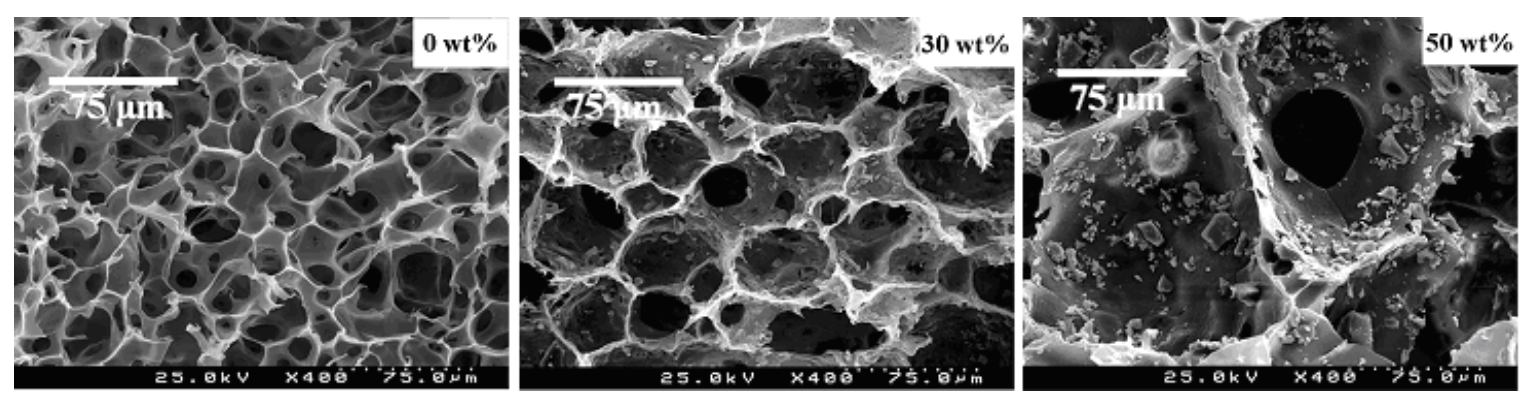

Fig. 1. SEM images of PLLA/BG scaffolds with different BG content.

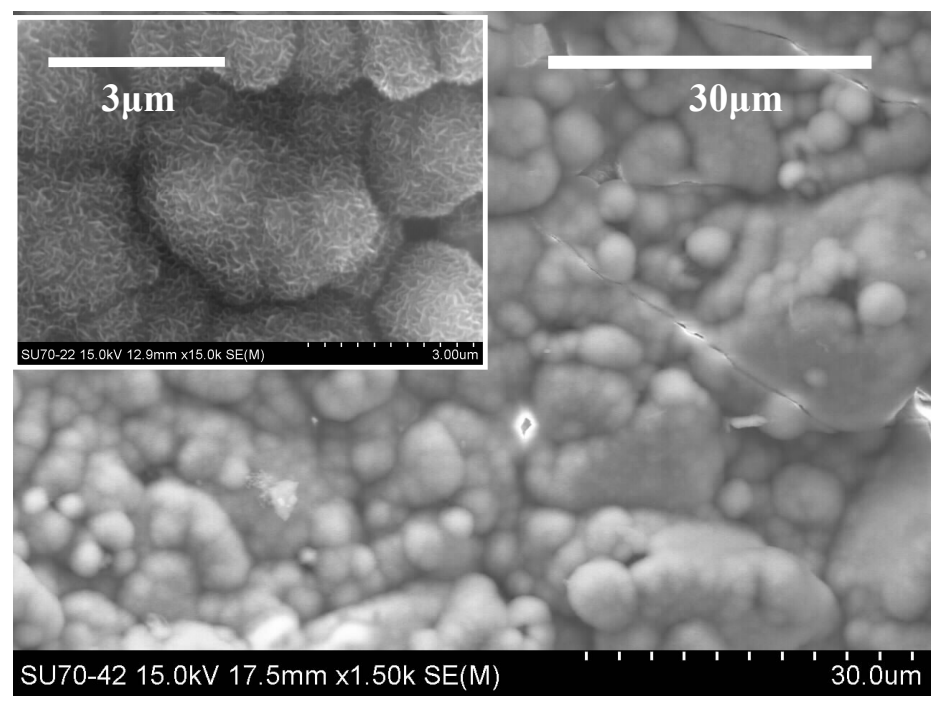

Fig. 2. SEM image of PLLA $/ 50 \% B G$ composite after immersion in SBF for 4 days.

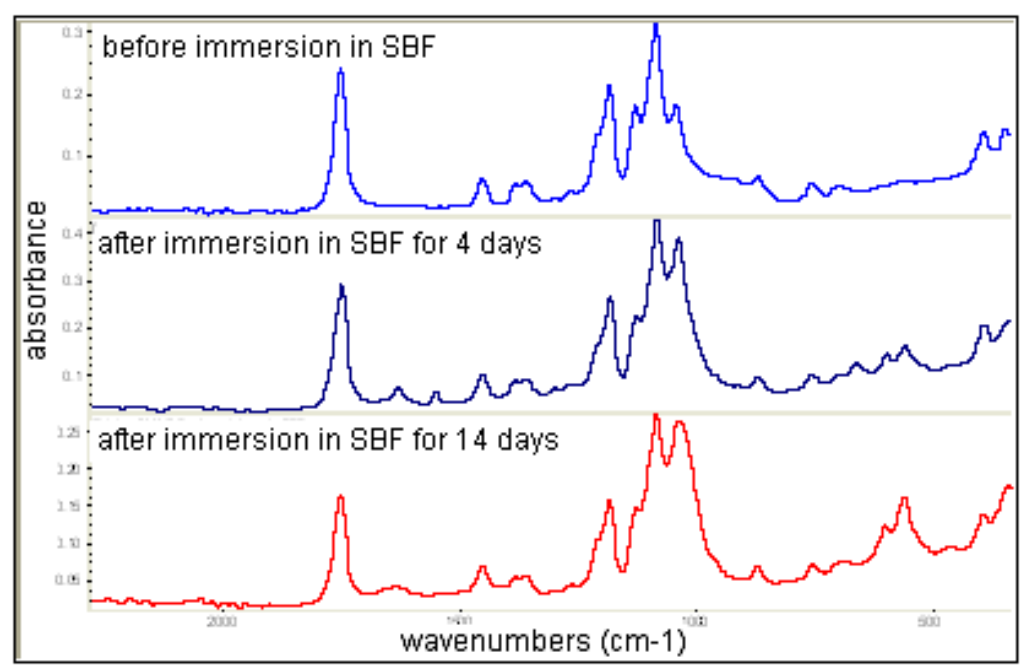

Fig. 3. FTIR spectra of PLLA/50\%BG composite before and after immersion in SBF. 